\title{
A database of associative strengths from the strength-sampling model: A theory-based supplement to the Nelson, McEvoy, and Schreiber word association norms
}

\author{
WILLIAM S. MaKI \\ Texas Tech University, Lubbock, Texas
}

\begin{abstract}
The strength-sampling model of free association (Nelson, McEvoy, \& Dennis, 2000) claims that the probability of word association in free-association norms results from a sampling process. For a given cue word, each response word has an underlying distribution of strength values. In the free-association task, presentation of the cue word activates a random sample of strengths, one for each response. The highest strength wins, and its response is reported. In the present work, gradient descent was used to compute the theoretical mean strengths for each cue-response pair in the Nelson, McEvoy, and Schreiber (2004) norms. The resulting database may be downloaded from www.psychonomic.org/archive/.
\end{abstract}

Understanding the relationships between words is a longstanding motivation for research and theory. Measures of possible associative and semantic relationships are prerequisites for that understanding. There are now several such measures. Relationships among words can be measured by analysis of large corpora of text (see, e.g., Kwantes, 2005; Landauer \& Dumais, 1997; Maki, McKinley, \& Thompson, 2004). Pairs of words can be rated for their association value (Koriat, Fiedler, \& Bjork, 2006; Maki, 2007) or their semantic similarity (e.g., Maki, Krimsky, \& Muñoz, 2006). The semantic relationships among words have been explored using a feature production technique (McRae, Cree, Seidenberg, \& McNorgan, 2005), but the oldest such measure is the conditional probability of word production in a free-association task (attributed to Galton; see Esper, 1973). Nelson, McEvoy, and Schreiber (2004) have published a large database containing such probabilities, and the theoretical basis for free-association probabilities is the target of the present report.

In the free-association task, participants are instructed to say (or write) the first (response) word that comes to mind given a cue word. In the Nelson et al. (2004) version of the procedure, a large number of participants (ranging from 94 to 206 per cue word) contributed responses. The conditional probability of a response word given a particular cue word defined the forward associative strength (FSG) binding the cue and the response. At present, the Nelson et al. (2004) norms contain distributions of responses and their probabilities for over 5,000 cue words.

As an example, consider the cue word CANARY. The most frequent response is BIRD (FSG $=.692$ ); the second, third, and fourth associates are YELLOW (FSG $=.111$ ), SING $(F S G=.032)$, and SONG (FSG $=.016)$. The FSGs sum to less than 1 (.851); idiosyncratic responses and failures to respond account for the remainder.

Associative strength is sometimes taken as a benchmark against which to evaluate other measures (see, e.g., Koriat et al., 2006; Maki, 2007). But it should be understood that associative strength is just another measure of relations among words in memory. Given status as a dependent variable, associative strength itself stands in need of explanation. As Nelson, McEvoy, and Dennis (2000) put it, "probability of free association provides a manifestation of strength rather than strength itself" (p. 897). The task then becomes one of theoretically specifying strength and how it underlies free-association probabilities.

Nelson et al. (2000) devised a model of free association that they called the relative strength hypothesis. The model stipulates that the associative strength of each cueresponse pair actually is a distribution of strengths. The variability reflects differences among items and individuals. The distribution of each cue-response pair was assumed to be normal and centered at some mean strength; the standard deviation was assumed (for convenience) to be 1.0. In the free-association task, presentation of a cue activates a set of strengths, one randomly drawn from each distribution associated with that cue. The strengths then compete, and the response corresponding to the highest value is the winner of the competition and is selected for output. The model here will be called the strengthsampling model because the set of associative strengths activated by a cue word is a random sample of strengths. 
The model is illustrated in the top panel (A) of Figure 1 using the cue word CANARY. The four normal distributions correspond, from left to right, to the four associates SONG, SING, YELLOW, and BIRD. The vertical line represents a response criterion (set to zero by Nelson et al., 2000). The separation of the distributions was determined by the computational procedures described below and used by Nelson et al. (2000). Note that, when drawing a randomly determined strength from each distribution, sometimes the values drawn from the leftmost (weaker strength) distributions will exceed the response criterion and exceed the value drawn from the rightmost (strongest strength) distribution. In this way, YELLOW, SING, and SONG will sometimes win out over BIRD.

Nelson et al. (2000) used the strength-sampling model to compute the means of the strength distributions and to use those computations to model the probabilities of secondresponse production in a two-response free-association procedure. They reported that "an optimization procedure was used to estimate the means of the strength distributions" (p. 893). It turns out that Nelson et al. (2000) recovered the means of the strength distributions by the optimization technique known as gradient descent (D. L. Nelson, personal communication, March 27, 2006). The theoretical means were computed by Nelson et al. (2000) just on the words used in their study. The computational effort described below was intended to provide the theoretical means for the entire Nelson et al. (2004) database.

\section{Computation of Mean Strengths via Gradient Descent}

Using gradient descent to discover mean strengths begins with drawing a randbm sample from each strength distribution for a given cue word. For each of many such samples, the winning response is that with the highest strength. The probabilities of the various responses, $p\left(R_{i}\right)$, are then compared with the free-association probabilities (FSGs) known from the association norms for that cue. The values of the mean strengths, $\mu_{i}$, are then adjusted proportionally to the error, $\mathrm{FSG}_{i}-p\left(R_{i}\right)$, and the entire procedure is repeated until the error is at a minimum. In the computations described here, the $\mu_{\mathrm{i}}$ values were computed for each cue word in the Nelson et al. (2004) word association norms. ${ }^{1}$ Following are the details of the algorithm used to discover $\mu_{i}$ values.

1. The forward associative strength for each response word was retrieved from the Nelson et al. (2004) norms.

2. $\mu_{i}$ was initialized, such that each $\mu_{i}=0$.

3. For each response, a random value $R_{i}$ was drawn from its distribution $\left(\mu_{i}, \sigma=1.0\right)$.

4. The winning response was that with the highest $R_{i}$.

5. Steps 3 and 4 were repeated 1,000 times to establish a distribution of the probabilities $p\left(R_{i}\right)$.

6 . The difference between the known and predicted probabilities (error), $\delta_{i}=\mathrm{FSG}_{i}-p\left(R_{i}\right)$, was computed for each response.

7. Each $\mu_{i}$ was adjusted by a fraction ( $\varepsilon$, the learning rate) of that error, $\Delta \mu_{i}=\varepsilon \cdot \delta_{i}$. In these computations, $\varepsilon=0.50$.
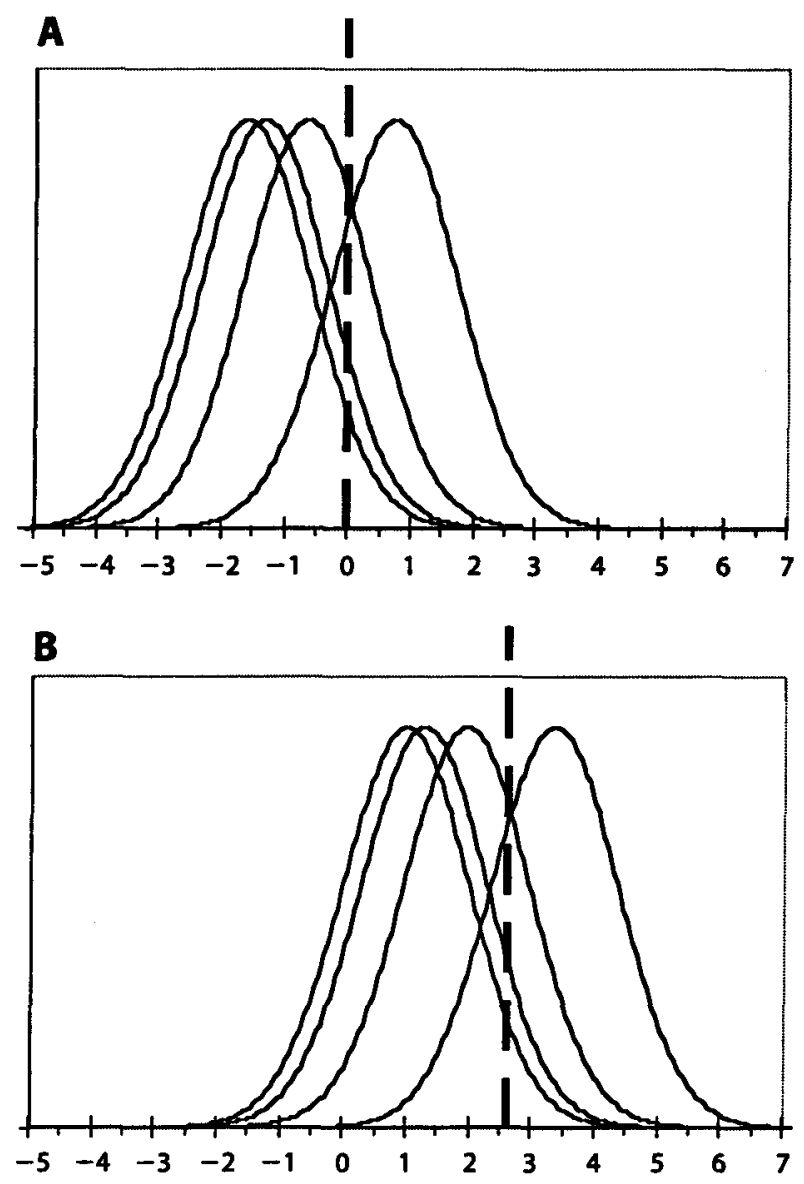

Figure 1. Ilustration of the strength-sampling model for the associates of CANARY. From left (weak) to right (strong), the associates are SONG, SING, YELLOW, and BIRD. In the original Nelson, McEvoy, and Dennis (2000) procedure, the response criterion was set at zero for all cue words (as in panel $A$ here). In the revised procedure described in the text, the response criterion was constrained to result in values greater than zero (as was done for panel B).

8. The quantity to be minimized was the root-mean square error, RMSE (i.e., the square root of the average of the squared $\delta_{i}$ values).

9. Steps 3-8 were repeated for at least 200 iterations and then continued until RMSE $<.005$. Over all cue words, the number of iterations averaged 212 (range: 200-483). The RMSE averaged .004 (range: .0008-.005).

10. Steps 2-9 were repeated for each cue word.

The present computations differed from those of Nelson et al. (2000) in two ways. First, in the present computations, $\mu_{i}$ was constrained to be positive so as to avoid the problems with roots of negative numbers in future modeling efforts. One other source of error was included in the computation of $\delta_{i}$ at Step 6-namely, the difference between a theoretical minimum strength $(+1.0)$ and the computed minimum $\mu_{i}$. The effect of this additional error was to force all the $\mu_{i}$ to be positive. The original Nelson et al. (2000) procedure placed no such constraint on their $\mu_{i}$, so $\mu_{i}$ could be negative. 
Second, the response criterion varied across cue sets. The response criterion is the minimum value of $R_{i}$ required for a response to be counted. When $R_{i}$ fell below the criterion, the response was regarded as idiosyncratic or as a nonresponse during free association. Nelson et al. (2000) set the response criterion at zero. In the present computations, the gradient descent program discovered solutions faster if the response criterion was allowed to vary. The criterion for each cue word was discovered by gradient descent simultaneously with the steps involved in the discovery of the $\mu_{i}$ values described above. ${ }^{2}$ For each run of the algorithm $(N=1,000$; Step 5), the proportion of idiosyncratic responses or nonresponses was computed, $p_{\text {residual }}=1-\Sigma p\left(R_{i}\right)$. This residual probability was compared with the corresponding probability obtained from the Nelson et al. (2004) database, $\mathrm{FSG}_{\text {residual }}=1-$ $\Sigma \mathrm{FSG}_{i}$, and the error signal used to adjust the response criterion was $\mathrm{FSG}_{\text {residual }}-p_{\text {residual }}$.

To assess the consequences of these changes in computational procedures, the mean strengths were computed for 90 cue words used in Maki's (2007) Experiment 3 using both the original Nelson et al. (2000) procedure and the adaptation presented here that constrained the minimum $\mu_{i}$ to be positive and allowed the response criterion to vary. The correlations between the $\mu_{i}$ values obtained by the original and modified procedures for the first three associates of each cue were all reliable, $r \mathrm{~s}=.95, .85$, and .81 , respectively. Thus, it appears that both procedures produce nearly identical results.

Further analysis showed that the response criterion for a cue word is independent of the differences among the $\mu_{i}$ values for that cue. Figure 1B shows the strength distributions and response criterion for CANARY. The separations of the distributions in Figure 1B are indistinguishable from those in Figure 1A. The values in Figure 1B are just shifted upward. All of the $\mu_{i}$ values in the 90-word set were adjusted by subtracting their corresponding response criteria, thus resetting the criteria to zero. These adjusted $\mu_{i}$ values were then used in regression analyses to predict those values found with the original Nelson et al. (2000) procedure for the first three associates of each cue. Intercepts averaged .036 , slopes averaged .999 , and these fits were excellent (average $R^{2}=.995$ ). Thus, both the original Nelson et al. (2000) procedure and the modification used here discover the same $\mu_{i}$ values when the response criteria are equated. ${ }^{3}$

The database has already proven of use. In a previous attempt to theoretically connect judged association to freeassociation norms, strength distributions were based on presumed patterns of lexical co-occurrence (Maki, 2007). In simulation work currently in progress (Maki, 2006), an alternative model of associative judgments relies on the empirically grounded distributions from the strength-sampling model. In other (unpublished) research conducted in collaboration with Marissa Krimsky, participants rank-ordered four associates of each cue word. We found that the distributions of rank orders computed from the strength-sampling model matched closely the distributions of ranks assigned by the human participants. As these examples indicate, the theoretical supplement to the association norms described here will enable the use of theoretical mean strengths for stimulus selection in future experiments and will be a useful starting point for future efforts to model the various measures of associative and semantic relationships.

The database and supporting software (MATLAB and Perl scripts) can be obtained from the Psychonomic Society's Norms, Stimuli, and Data archive.

\section{AUTHOR NOTE}

Correspondence related to this article may be sent to W. S. Maki, Department of Psychology, Texas Tech University, Lubbock, TX 79409 (e-mail: bill.maki@ttu.edu).

\section{REFERENCES}

ESPER, E. A. (1973). Analogy and association in linguistics and psychology. Athens, GA: University of Georgia Press.

KorIAT, A., Fiedler, K., \& BJoRK, R. A. (2006). The inflation of conditional predictions. Journal of Experimental Psychology: Genenal, 135, 429-447.

Kwantes, P. J. (2005). Using context to build semantics. Psychonomic Bulletin \& Review, 12, 703-710.

LANDAUER, T. K., \& Dumais, S. T. (1997). A solution to Plato's problem: The latent semantic analysis theory of acquisition, induction, and representation of knowledge. Psychological Review, 104, 211-240.

MAKI, W. S. (2006, November). Ratings of nondominant associations: Implications for models of associative judgment. Paper presented at the meeting of the Psychonomic Society, Houston, TX.

MAK, W. S. (2007). Judgments of associative memory. Cognitive Psychology, 54, 319-353.

MAKI, W. S., KRIMSKY, M., \& MUÑoz, S. (2006). An efficient method for estimating semantic similarity based on feature overlap: Reliability and validity of semantic feature ratings. Behavior Research Methods, 38, 153-157.

Maki, W. S., McKinley, L. N., \& Thompson, A. G. (2004). Semantic distance norms computed from an electronic dictionary (WordNet). Behavior Research Methods, Instruments, \& Computers, 36, 421-431.

McRae, K., Cree, G. S., Seidenberg, M. S., \& McNorgan, C. (2005). Semantic feature production norms for a large set of living and nonliving things. Behavior Research Methods, 37, 547-559.

Nelson, D. L., MCEvoy, C. L., \& DenNis, S. (2000). What is free association and what does it measure? Memory \& Cognition, 28, 887-899.

Nelson, D. L., McEvoy, C. L., \& Schreiber, T. A. (2004). The University of South Florida free association, rhyme, and word fragment norms. Behavior Research Methods, Instruments, \& Computers, 36, 402-407.

\section{NOTES}

1. The solution for one cue word, LEFT, could not be determined because only one response is listed in the norms. The probabilities for another word, BARTENDER, are at odds with the frequencies published in the database; the free-association probabilities for that cue set were recomputed on the basis of the frequencies.

2. Subtracting the response criterion from the computed mean strengths centers each distribution at zero, the Nelson et al. (2000) response criterion.

3. In the sample of 90 pairs, the response criterion was correlated with cue set size, $r=.66$. This correlation needs to be interpreted cautiously, because the correlation between response criterion and cue set size for all 5,018 words in the database is only $r=.178$.

\section{ARCHIVED MATERIALS}

The following materials may be accessed through the Psychonomic Society's Norms, Stimuli, and Data archive, www.psychonomic.org/archive/. 
To access these files, search the archive for this article using the journal (Behavior Research Methods), the author's name (Maki), and the publication year (2008).

FILE: Maki-BRM-2008.zip

DESCRIPTION: The compressed archive file contains six files: mudb.txt is a space-delimited file containing 5,018 lines. Each line contains a cue word, the number of iterations required for gradient descent, the stopping error, the response criterion, and all the response words each followed by its computed mean strength.

nmd.m is the MATLAB script that manages input/output and calls the two other scripts. fsgreader.m reads in a file of cue and response words and their FSG values.

gd2.m performs the gradient descent.

get-all-cue-sets.pl and reorder-mudb.pl are two Peri scripts that format the input to and output from the MATLAB scripts.

The zip file also may be downloaded from ftp://ftp.ttu.edu/pub/maki.

AUTHOR's E-MAIL ADDRESS: bill.maki@ttu.edu.

(Manuscript received November 2, 2006; revision accepted for publication March 20, 2007.) 